\title{
Depressão como fator de risco de morbidade imediata e tardia pós-revascularização cirúrgica do miocárdio
}

\author{
Depression as a risk factor for early and late morbidity after coronary artery bypass surgery
}

Fábio Augusto PINTON, Cecília Freire de CARVALHO, Maria Cristina de Oliveira Santos MIYAZAKI, Moacir Fernandes de GODOY

\section{Resumo}

Objetivo: Avaliar a presença de sintomas de depressão no pré-operatório, no pós-operatório imediato (POI) e pósoperatório tardio (POT) de pacientes com indicação eletiva de cirurgia de revascularização do miocárdio e seu impacto na morbidade pós-operatória imediata e tardia.

Método: Cinqüenta e oito pacientes internados na Enfermaria de Cirurgia Cardíaca para realização de operação eletiva de revascularização do miocárdio responderam ao Inventário de Depressão de Beck, antes da operação (fase I), antes da alta hospitalar (fase II) e três meses após a alta (fase III). A média de idade dos pacientes foi 61,2 (34 a 78 anos; DP:10,1), 34 (58,6\%) eram homens, $31(55,4 \%)$ com infarto prévio, 35 (62,5\%) com fração de ejeção maior que $40 \%$ e 19 (33\%) diabéticos.

Resultados: Doze $(20,7 \%)$ pacientes apresentaram sintomas de depressão na fase I, 13 (23,6\%), na II e 4 (9,8\%), na III. Dezoito $(31,0 \%)$ pacientes apresentaram complicações no POI, 17 (34,0\%), no POT. Complicações no POI ocorreram com maior freqüência em pacientes mais velhos (idade superior a 65 anos; $p=0,003)$, com pelo menos três vasos revascularizados $(p=0,001)$ e com depressão na fase $I(p=0,011)$. Quando estas variáveis foram associadas às complicações presentes no POT, houve significância para o sexo feminino $(p=0,006)$ e depressão na fase II $(p=0,008)$. Pacientes do sexo feminino apresentaram mais sintomas de depressão durante a internação $(\mathbf{p}=\mathbf{0 , 0 4})$.

Conclusão: Idade superior a 65 anos, sexo feminino, pelo menos três vasos revascularizados e sintomas de depressão durante a internação mostraram-se associados a maior número de complicações no pós-operatório de cirurgia de revascularização do miocárdio. Pacientes submetidos à cirurgia de revascularização do miocárdio devem ser avaliados em relação à depressão e tratados se necessário, uma vez que esta pode estar associada a complicações no pós-operatório.

Descritores: Coronariopatia. Depressão. Revascularização miocárdica.

\footnotetext{
Trabalho realizado na Faculdade de Medicina de São José do Rio Preto (FAMERP); Hospital de Base da Fundação Faculdade Regional de Medicina (FUNFARME).

Endereço para correspondência:

Fábio Augusto Pinton. Av. Brigadeiro Faria Lima, 5416. Bairro São Pedro - São José do Rio Preto, SP, Brasil. CEP 15090-000 - Tel (17) 3201-5700 ramal 5842

E-mail: fapinton@yahoo.com.br
}

Artigo recebido em novembro de 2005 Artigo aprovado em fevereiro de 2006 


\section{Abstract}

Objective: To assess presence of symptoms of depression in the in the preoperative period, immediate postoperative period (IPP) and in the late postoperative period (LPP) in patients with coronary artery disease undergoing bypass surgery and its impact on early and late postoperative morbidity.

Method: Fifty-eight inpatients waiting to undergo an elective bypass surgery completed the Beck Depression Inventory (BDI) before surgery (Period I), after surgery just before hospital discharge (Period II) and three months later (Period III). Patients mean age was 61.2 (34 to 78 years; SD: 10.1), $34(58.6 \%)$ were male, $31(55.4 \%)$ had a history of infarction, $35(62.5 \%)$ had ejection fraction $>40 \%$ and 19 $(33.0 \%)$ had diabetes.

Results: Depression symptoms were identified in 12 (20.7\%) patients in Period I, 13 (23.6\%) in Period II and four $\mathbf{( 9 . 8 \% )}$ in Period III. Eighteen $(31.0 \%)$ patients had

\section{INTRODUÇÃO}

Pesquisas sobre doença arterial coronariana (DAC) e o comportamento começaram a ser realizadas quando estudos epidemiológicos identificaram inúmeros fatores ambientais e associados ao estilo de vida envolvidos na etiologia e na patogênese da doença. Com o avanço do conhecimento na área, evidências começaram a sugerir que "reconhecer e tratar o estresse psicossocial em pacientes com DAC pode reduzir a morbidade e a mortalidade subseqüentes” [1].

Do ponto de vista psicossocial, algumas variáveis foram identificadas como possíveis fatores de risco para o desenvolvimento e para a progressão da doença, como estresse agudo e crônico, hostilidade, depressão, suporte social e status socioeconômico [1].

A depressão está associada ao risco para doenças cardiovasculares, independente dos fatores clássicos de risco, tanto para pacientes saudáveis, como para aqueles que apresentam DAC [2-5]. Entre os pacientes com DAC, o risco de mortalidade cardíaca é de duas a quatro vezes maior naqueles que apresentam depressão [6]. Dentre os mecanismos fisiológicos possivelmente associados à depressão e ao aumento do risco cardiovascular, estão a disfunção endotelial, a desregulação do sistema nervoso autônomo e do eixo hipotálamo-hipófise-adrenal e a hipercoagulabilidade sangüínea [6,7].

Diversos estudos realizados na última década têm documentado aumento na prevalência de depressão entre pacientes com várias manifestações da DAC. A prevalência de depressão maior, como definida pelo DSM-IV [8], varia complications in the IPP, $17(34.0 \%)$ in the LPP. IPP complications were more frequent for older patients (more than 65 years; $p=0.003)$, with at least three grafts $(p=0.001)$ and depression in Period $I(p=0.011)$. When those variables were associated with complications on the LPP, there was a significant difference for females $(p=0.006)$ and depression in Period II $(p=0.008)$. Female patients had more depression symptoms while staying in hospital $(p=0.04)$.

Conclusion: More than 65 years, females, three or more grafts and depression symptoms in the postoperative period were associated with more complications after bypass surgery. Patients undergoing bypass surgery should be carefully monitored for depression and treated if necessary since it may be associated with complications after surgery.

Descriptors: Coronary disease. Depression. Myocardial revascularization.

entre $16 \%$ e $23 \%$ e a de altos níveis de sintomas depressivos entre 31,5\% e 60\% [5,9]. Além disso, a depressão prediz importantes eventos cardíacos futuros [3, 5, 9,10].

A associação entre depressão e prognóstico de pacientes com DAC submetidos à cirurgia de revascularização tem sido enfocada em alguns estudos, cujos resultados indicam a necessidade de identificar e manejar adequadamente aqueles com risco aumentado para depressão $[11,12]$. Blumenthal et al. [13] avaliaram 817 pacientes submetidos a este procedimento e concluíram que, apesar dos avanços clínicos e cirúrgicos, "a depressão é um importante preditor independente de morte após a cirurgia de revascularização e deve ser cuidadosamente monitorada e tratada quando necessário".

Considerando a freqüência com que a cirurgia de revascularização tem sido realizada e o risco de complicações associadas à depressão, foram objetivos deste estudo: avaliar a presença de sintomas de depressão no pré e pósoperatório imediato e tardio de pacientes com indicação eletiva de cirurgia de revascularização do miocárdio e seu impacto na morbidade pós-operatória imediata e tardia.

\section{MÉTODO}

Após terem sido informados sobre o estudo, concordarem em participar e assinarem um termo de consentimento livre e esclarecido, 58 pacientes com DAC, internados na Enfermaria de Cirurgia Cardíaca e com indicação de cirurgia eletiva de revascularização do miocárdio, foram incluídos no estudo. 
Os pacientes responderam ao Inventário Beck de Depressão* [14,15], antes da operação (fase I), antes da alta hospitalar (fase II) e três meses depois (fase III). O Inventário Beck de Depressão é uma escala de 21 itens, que avalia presença e gravidade de sintomas de depressão. Cada item é graduado em uma escala de 0 a 3 , o escore total pode variar entre 0 e 63 pontos e os resultados estão significativamente associados a avaliações clínicas de depressão. Tem sido amplamente utilizado com pacientes cardíacos e as evidências sugerem que o risco para futuros eventos cardíacos está associado com a magnitude dos sintomas depressivos. A versão brasileira do instrumento foi aprovada pelo Sistema de Avaliação de Testes Psicológicos / Conselho Federal de Psicologia.

Foram analisadas as características da amostra para alguns fatores de risco, além de quadro prévio de depressão, classe social, segundo classificação da ABIPEME [16], fração de ejeção prévia à operação com nível de corte maior ou igual a $40 \%$ pelo ecocardiograma ou disfunção ventricular esquerda de grau leve ou normal pelo estudo hemodinâmico. As complicações analisadas no pós-operatório foram divididas em cardiológicas (arritmias e infarto documentados), renais (aumento da creatinina em níveis superiores a $2 \mathrm{mg} / \mathrm{dL}$ ), neurológicas (acidente vascular cerebral, acidente isquêmico transitório), infecciosas (qualquer infecção com necessidade de utilização de antibióticos) e outras (derrame pleural, trombose venosa, deiscência de sutura). Foi considerado pós-operatório imediato (POI) o período entre o término da operação e a alta hospitalar e pós-operatório tardio (POT), o período entre a alta hospitalar até três meses depois. Estes dados foram obtidos por meio de revisão dos prontuários dos pacientes.

Foram utilizados na análise estatística teste quiquadrado, teste Mood para medianas e regressão logística, sendo considerado significante um valor $\mathrm{p}<0,05$.

\section{RESULTADOS}

A idade dos pacientes variou entre 34 e 78 anos (média de idade 61,2; DP:10,1) e 34 (58,5\%) eram do sexo masculino. A Tabela 1 mostra que $75,4 \%$ da amostra não apresentava história de depressão e 80\%, de tabagismo. Uma análise das características de saúde destes pacientes evidenciou que 35 (62,5\%) tinham boa fração de ejeção, 31 (55,4\%) haviam sofrido infarto e 19 (33,0\%) eram diabéticos. Com relação à classe social, 29 (50,0\%) pacientes eram da classe D, 22 (37,9\%) da classe C, 5 (8,6\%) da classe A ou B e 2 (3,4\%) da classe E.

O tempo médio de internação antes da operação foi 6,2 dias (DP 6,2; Mediana:4) e após a operação de 8,5 dias (DP 5,2; Mediana:7). Onze (18,9\%) pacientes ficaram internados cinco dias ou menos durante o pós-operatório.

Complicações no POI ocorreram em 18 (31\%) pacientes:
12 complicações cardíacas, 12 infecciosas, cinco renais e três mortes. No POT, 17 (34\%) tiveram complicações: 13 infecciosas, duas cardíacas, uma renal, uma neurológica e três outras. Para 5 (9,1\%) pacientes não foi possível avaliar as complicações no POT, uma vez que estes ainda não completaram os três meses necessários para a avaliação pós-operatória (fase III).

Na fase I (pré-cirurgia), 12 (20,7\%) pacientes obtiveram escore de depressão maior ou igual a 10 (10 em grau leve e dois em grau moderado). Na fase II (antes da alta hospitalar), $13(23,6 \%)$ pacientes obtiveram escore superior a 10 (oito em grau leve e cinco em grau moderado). Na fase III (três meses após a alta), quatro (9,8\%) pacientes apresentaram sintomas de depressão, três em grau leve e um, grave. Não foi possível realizar o inventário na fase III em 14 (25,5\%) pacientes, pelo não comparecimento dos mesmos para acompanhamento ambulatorial.

Na análise de regressão logística para as variáveis e os sintomas de depressão na fase I ou II, houve significância estatística em relação ao sexo feminino ( $p=0,04 \mathrm{OR}=3,25 \mathrm{IC}$ 1,05-10,02).

Na análise das variáveis com a presença de complicações no POI, houve significância estatística em relação à idade maior ou igual a 65 anos $(p=0,003)$, número de enxertos maior ou igual a $3(\mathrm{p}=0,001)$ e sintomas de depressão na fase I $(\mathrm{p}=0,011)$. Já em relação à presença de complicações no POT, houve significância entre sexo feminino $(p=0,006)$ e sintomas de depressão na fase II $(\mathrm{p}=0,008)$. Além disso, os pacientes que apresentaram sintomas de depressão na fase I tiveram maior número de complicações no POI $(\mathrm{p}=0,003)$ e aqueles que apresentaram sintomas na fase II tiveram maior número de complicações no POT (p=0,002) - Figuras 1 e 2.

Neste estudo, houve associação entre sintomas de depressão e complicações no pós-operatório de cirurgia de revascularização do miocárdio. Os pacientes que apresentaram sintomas de depressão na fase I tiveram mais complicações no POI (cardiológicas, infecciosas e renais), enquanto que aqueles que apresentaram tais sintomas na fase II tiveram mais complicações no POT (cardíacas, infecciosas, renal, neurológica, entre outras).

Além da depressão, três outros fatores tiveram associação com complicações pós-operatórias: idade igual ou superior a 65 anos e número de enxertos maior ou igual a 3 estiveram associados às complicações no POI e o sexo feminino, às complicações no POT. Neste estudo não houve diferença entre as classes A, B e C comparadas com a D e E. Porém, quando foram agrupadas apenas as classes sociais A e B (8,6\%), os pacientes não apresentaram nenhuma complicação no POI e no POT, nem sintomas de depressão.

Dos três óbitos da amostra, apenas um apresentava sintomas de depressão na fase I, não havendo portanto significância estatística. 
Tabela 1. Relação entre variáveis analisadas, depressão e complicações no pós-operatório imediato (POI) e tardio (POT).

\begin{tabular}{|c|c|c|c|c|}
\hline & Número de pacientes & IBD = 10 na fase I ou II & Complicações POI & Complicações POT \\
\hline \multicolumn{5}{|l|}{ idade } \\
\hline$<65$ anos & $32(55,2 \%)$ & $11(34,4 \%)$ & $4(12,5 \%)^{*}$ & $10(34,5 \%)$ \\
\hline$>65$ anos & $26(44,8 \%)$ & $9(34,6 \%)$ & $14(53,8 \%)^{*}$ & $7(33,3 \%)$ \\
\hline \multicolumn{5}{|l|}{ sexo } \\
\hline masculino & $34(58,6 \%)$ & $8(23,5 \%)^{*}$ & $8(23,5 \%)$ & $5(16,7 \%)^{*}$ \\
\hline feminino & $24(41,4 \%)$ & $12(50,0 \%)^{*}$ & $10(41,7 \%)$ & $12(60,0 \%)^{*}$ \\
\hline \multicolumn{5}{|l|}{ história de depressão } \\
\hline não & $43(75,4 \%)$ & $13(30,2 \%)$ & $11(34,4 \%)$ & $13(35,1 \%)$ \\
\hline $\operatorname{sim}$ & $14(24,6 \%)$ & $7(50,0 \%)$ & $6(42,9 \%)$ & $4(30,8 \%)$ \\
\hline \multicolumn{5}{|l|}{ tabagismo } \\
\hline não & $44(80,0 \%)$ & $16(36,4 \%)$ & $14(31,8 \%)$ & $13(34,2 \%)$ \\
\hline $\operatorname{sim}$ & $11(20,0 \%)$ & $3(27,3 \%)$ & $3(27,3 \%)$ & $4(40,0 \%)$ \\
\hline \multicolumn{5}{|l|}{ diabetes } \\
\hline não & $39(67,2 \%)$ & $12(30,8 \%)$ & $9(23,1 \%)$ & $11(31,4 \%)$ \\
\hline $\operatorname{sim}$ & $19(32,8 \%)$ & $8(42,1 \%)$ & $9(47,4 \%)$ & $6(40,0 \%)$ \\
\hline \multicolumn{5}{|l|}{ história de IAM } \\
\hline não & $25(44,6 \%)$ & $8(32,0 \%)$ & $8(32,0 \%)$ & $7(35,0 \%)$ \\
\hline $\operatorname{sim}$ & $31(55,4 \%)$ & $12(38,7 \%)$ & $10(32,3 \%)$ & $10(35,7 \%)$ \\
\hline \multicolumn{5}{|l|}{ Classe social } \\
\hline A, B ou C & $27(46,6 \%)$ & $7(25,9 \%)$ & $6(22,2 \%)$ & $5(22,7 \%)$ \\
\hline D ou E & $31(53,4 \%)$ & $13(41,9 \%)$ & $12(38,7 \%)$ & $12(42,9 \%)$ \\
\hline \multicolumn{5}{|l|}{ mora sozinho } \\
\hline não & $53(93,0 \%)$ & $19(35,8 \%)$ & $15(28,3 \%)$ & $17(36,2 \%)$ \\
\hline $\operatorname{sim}$ & $4(7,0 \%)$ & $1(25,0 \%)$ & $2(50,0 \%)$ & 0 \\
\hline \multicolumn{5}{|l|}{ fração de ejeção } \\
\hline > 40\% ou disfunção leve & $35(62,5 \%)$ & $13(37,1 \%)$ & $13(37,1 \%)$ & $10(33,3 \%)$ \\
\hline$<40 \%$ ou moderada/grave & $21(37,5 \%)$ & $7(33,3 \%)$ & $4(19,1 \%)$ & $7(35,0 \%)$ \\
\hline \multicolumn{5}{|l|}{ número de enxertos } \\
\hline 1 ou 2 & $34(60,7 \%)$ & $10(29,4 \%)$ & $5(14,7 \%)^{*}$ & $10(33,3 \%)$ \\
\hline 3 ou mais & $22(39,3 \%)$ & $10(45,5 \%)$ & $13(59,1 \%)^{*}$ & $7(36,8 \%)$ \\
\hline \multicolumn{5}{|l|}{ IBD na fase I } \\
\hline$<10$ & $46(79,3 \%)$ & - & $10(21,7 \%)^{*}$ & - \\
\hline$>10$ & $12(20,7 \%)$ & - & $8(66,7 \%)^{*}$ & - \\
\hline \multicolumn{5}{|l|}{ IBD na fase II } \\
\hline$<10$ & $42(76,4 \%)$ & - & - & $8(21,6 \%)^{*}$ \\
\hline$>10$ & $13(23,6 \%)$ & - & - & $9(69,2 \%)^{*}$ \\
\hline
\end{tabular}

* p<0,005. IBD - Inventário Beck de Depressão (escore de depressão); IAM - Infarto Agudo do Miocárdio 


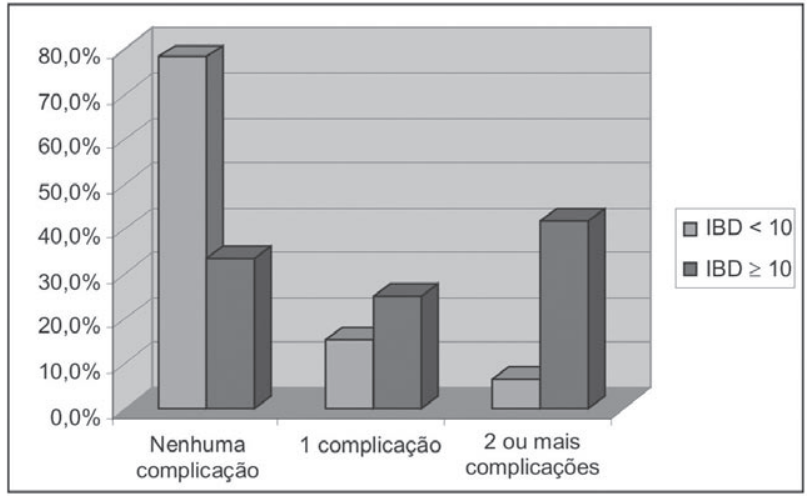

Fig. 1 - Escore no Inventário Beck de Depressão na fase I e número de complicações no pós-operatório tardio $(p=0,003)$

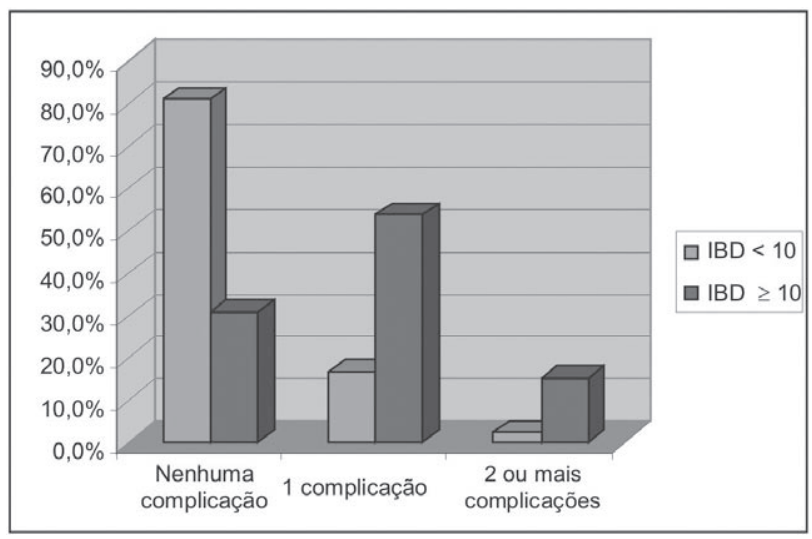

Fig. 2 - Escore no Inventário Beck de Depressão na fase II e número de complicações no pós-operatório tardio $(p=0,002)$

\section{DISCUSSÃO}

Em relação à idade e ao sexo da amostra, os dados obtidos são compatíveis com a literatura, isto é, houve predominância de pacientes mais idosos e do sexo masculino submetidos à cirurgia de revascularização do miocárdio [6,11-13,17].

Uma análise das condições de saúde dos pacientes indica que estas também são compatíveis com a literatura [6,12,13,17]. Diabetes, tabagismo, infarto prévio e depressão, condições identificadas em parte dos pacientes da amostra, estão associados à doença arterial coronária [2-5].

A predominância de pacientes das classes socioeconômicas $\mathrm{C}$ e D corresponde à realidade dos pacientes atendidos na instituição onde foi realizado o estudo.
Um período mais longo de internação para os pacientes da amostra foi observado, quando comparado a um único estudo identificado na literatura, analisando período de internação [17]. É possível que um período mais longo de internação para os pacientes deste estudo esteja relacionado a questões sociais, como falta de serviço médico adequado próximo à residência dos pacientes ou a necessidade de medicações de alto custo no pós-operatório, que não estariam disponíveis nas unidades básicas de saúde.

Complicações pós-operatórias, observadas em 18\% dos pacientes da amostra estudada, ressaltam a necessidade de cuidados especiais em relação a estes pacientes, embora algumas complicações possam realmente ocorrer.

Houve impossibilidade de avaliar 14 pacientes na fase III do estudo, uma vez que estes não realizaram o acompanhamento ambulatorial na instituição. O fato de grande parte dos pacientes atendidos na instituição serem provenientes de outras cidades, inclusive de outros estados, faz com que, após a operação, estes realizem o acompanhamento médico em sua cidade de origem.

Rymaszewska et al. [11] identificaram presença de sintomas de depressão em 32\% dos pacientes na fase I e em 28,3\%, na fase II, porém em maior número na fase III (26,4\%). É possível que esta diferença de resultado em relação à depressão dos pacientes deste estudo esteja relacionada à perda de alguns pacientes na fase III, à possibilidade dos pacientes terem sido encaminhados para tratamento da depressão ou, ainda, à melhora da qualidade de vida dos pacientes, reduzindo assim os sintomas de depressão. Outros estudos obtiveram prevalência de depressão, antes ou depois da operação, em torno de 20 a 25\% e 38\% [13,17].

A maior freqüência de depressão para o sexo feminino observada neste estudo é também um dado compatível com a literatura, que indica maior prevalência entre as mulheres $[6,11,13,18]$.

Neste estudo foi observada uma relação entre sintomas de depressão e complicações no pós-operatório. Trabalhos com seguimento superior a três meses também identificaram esta relação. Connerney et al. [17] observaram que 20\% dos pacientes com sintomas de depressão tiveram eventos cardíacos no período de 12 meses após a operação, sendo que apenas $11 \%$ dos que não apresentaram depressão tiveram este tipo de problema. Blumenthal et al. [13] encontraram freqüência duas vezes superior de eventos cardíacos em pacientes depressivos, comparados aos não depressivos, em 12 meses após operação.

Além da depressão e da idade superior a 65 anos, número de enxertos maior ou igual a 3 também se mostrou associado a complicações no pós-operatório imediato, enquanto que sexo feminino mostrou-se associado a complicações no pósoperatório tardio. Connerney et al. [17] relataram que sexo feminino e depressão maior foram preditores independentes de eventos cardíacos em 12 meses após operação. 
Segundo Kop [19] e Rozanski et al. [3], há uma forte evidência que baixo nível socioeconômico esteja associado ao risco de DAC e suas manifestações clínicas. Quando agrupados apenas os pacientes das classes A e B deste estudo, estes não apresentaram complicações no POI e POT, nem sintomas de depressão.

Não houve significância estatística entre o número de óbitos deste estudo e sintomas de depressão. Este dado é semelhante ao obtido por Connerney et al. [17], porém distinto do encontrado por Blumenthal et al. [13].

Algumas limitações do estudo devem ser apontadas como o curto tempo de seguimento dos pacientes, que talvez esteja associado ao menor número de complicações e mortes. A dificuldade encontrada por alguns pacientes para compreender o Inventário de Depressão, associada à baixa escolaridade, o tamanho reduzido da amostra, bem como a dificuldade em realizar o seguimento de todos os pacientes, podem também ser apontadas.

\section{CONCLUSÕES}

Sintomas de depressão acima do escore de corte utilizado foram identificados em aproximadamente 25\% da amostra.

Houve associação entre sintomas de depressão e complicações no pós-operatório. Outros fatores associados a complicações foram sexo feminino, idade maior que 65 anos e número de enxertos maior ou igual a três.

Embora o instrumento utilizado para avaliar depressão não forneça diagnóstico de transtorno de humor, é útil para identificar pacientes com sintomas em número suficiente para serem mais bem investigados com relação ao problema.

Pesquisas futuras são necessárias com maior número de pacientes e maior tempo de seguimento após a operação. Além disso, estudos comparando grupo que recebeu intervenção psicológica e/ou medicação para depressão com grupo controle seriam úteis para verificar a efetividade de fornecer atendimento em saúde mental para estes pacientes, concomitante ao atendimento convencional.

\section{AGRADECIMENTOS}

À Fundação de Apoio à Pesquisa do Estado de São Paulo - FAPESP pela bolsa de iniciação científica para Fábio Augusto Pinton. Ao CNPq, pela bolsa de produtividade em pesquisa para Maria Cristina O.S. Miyazaki. Ao professor José Antônio Cordeiro, livre docente em estatística pela UNESP, pela colaboração na análise estatística dos dados.

* Um escore de corte mais baixo foi utilizado para minimizar falsos negativos, conforme sugerido por Beck \& Steer, 1993[14]

\section{REFERÊNCIAS}

1. Krantz DS, McCeney MK. Effects of psychological and social factors on organic disease: a critical assessment of research on coronary heart disease. Annu Rev Psychol. 2002;53:341-69.

2. Pignay-Demaria V, Lespèrance F, Demaria RG, Frasure-Smith N, Perrault LP. Depression and anxiety and outcomes of coronary artery bypass surgery. Ann Thorac Surg. 2003;75(1):314-21.

3. Rozanski A, Blumenthal JA, Kaplan J. Impact of psychological factors on the pathogenesis of cardiovascular disease and implications for therapy. Circulation. 1999;99(16):2192-217.

4. Hemingway H, Malik M, Marmot M. Social and psychosocial influences on sudden cardiac death, ventricular arrhythmia and cardiac autonomic function. Eur Heart J. 2001;22(13):1082-101.

5. Musselman DL, Evans DL, Nemeroff CB. The relationship of depression to cardiovascular disease: epidemiology, biology, and treatment. Arch Gen Psychiatry. 1998;55(7):580-92.

6. Sherwood A, Hinderliter AL, Watkins LL, Waugh RA, Blumenthal JA. Impaired endothelial function in coronary heart disease patients with depressive symptomatology. J Am Coll Cardiol. 2005;46(4):656-9.

7. Lett HS, Blumenthal JA, Babyak MA, Sherwood A, Strauman $\mathrm{T}$, Robins $\mathrm{C}$ et al. Depression as a risk factor for coronary artery disease: evidence, mechanisms and treatment. Psychosom Med. 2004;66(3):305-15.

8. American Psychiatric Association. Manual diagnóstico e estatístico de transtornos mentais. DSM-IV-TR. $4^{\mathrm{a}}$ ed rev. Porto Alegre:ArtMed;2002.

9. Glassman AH, Shapiro PA. Depression and the course of coronary artery disease. Am J Psychiatry. 1998;155(1):4-11.

10. Lesperance F, Freasure-Smith N. Depression in patients with cardiac disease: a practical review. J Psychosom Res. 2000;48(4-5):379-91.

11. Rymaszewska J, Kiejna A, Hadrys T. Depression and anxiety in coronary artery bypass grafting surgery. Eur Psychiatry. 2003;18(4):155-60.

12. Borowicz Jr L, Royall R, Grega M, Selnes O, Lyketsos C, McKhann G. Depression and cardiac morbidity 5 years after coronary artery bypass surgery. Psychosomatics. 2002;43(6):464-71.

13. Blumenthal JA, Lett HS, Babyak MA, White W, Smith PK, Mark BD et al. Depression as a risk factor for mortality after coronary artery bypass surgery. Lancet. 2003;362(9384):604-9. 
14. Beck AT, Steer RA. Beck depression inventory. Manual. San Antonio, Texas: Psychological Corporation;1993.

15. Cunha JA. Manual da versão em português das escalas Beck. São Paulo:Casa do Psicólogo;2001.

16. ABIPEME - Associação Brasileira dos Institutos de Pesquisa de Mercado. Proposição para um novo critério de classificação socioeconômica, 1978. São Paulo:Mímeo;1978. 15p.

17. Connerney I, Shapiro PA, McLaughlin JS, Bagiella E, Sloan RP. Relation between depression after coronary artery bypass surgery and 12-month outcome: a prospective study. Lancet. 2001;358(9295):1766-71.

18. Ariyo AA, Haan M, Tangen CM, Rutledge JC, Cushman M, Dobs A et al. Depressive symptoms and risks of coronary heart disease and mortality in elderly Americans. Cardiovascular Health Study Collaborative Research Group. Circulation. 2000;102(15):1773-9.

19. Kop WJ. Acute and chronic psychological risk factors for coronary syndromes: moderating effects of coronary artery disease severity. J Psychosom Res. 1997; 43(2):167-81. 